Marquette University

e-Publications@Marquette

Physics Faculty Research and Publications

Physics, Department of

$1-1-2009$

Dependence of Domain Wall Structure for Low

Field Injection into Magnetic Nanowires

Andrew Kunz

Marquette University, andrew.kunz@marquette.edu

Sarah C. Reiff

Marquette University

Published version. Applied Physics Letters, Vol. 94, No. 19 (2009). DOI. (C) 2009 American Institute of Physics. Used with permission. 


\title{
Dependence of domain wall structure for low field injection into magnetic nanowires
}

\author{
Andrew Kunz ${ }^{\mathrm{a})}$ and Sarah C. Reiff \\ Department of Physics, Marquette University, Milwaukee, Wisconsin 53233, USA
}

(Received 18 February 2009; accepted 28 April 2009; published online 14 May 2009)

\begin{abstract}
Micromagnetic simulation is used to model the injection of a domain wall into a magnetic nanowire with field strengths less than the so-called Walker field. This ensures fast, reliable motion of the wall [N. L. Schryer and L. R. Walker, J. Appl. Phys. 45, 5406 (1974)]. When the wire is located at the edge of a small injecting disk, a bias field used to control the orientation of the domain wall can reduce the pinning potential of the structure. The low field injection is explained by a simple model, which relies on the topological nature of a domain wall. The technique can quickly inject multiple domain walls with a known magnetic structure. (C) 2009 American Institute of Physics.
\end{abstract}

[DOI: 10.1063/1.3139066]

The motion of a domain wall through a long, narrow nanowire has been studied extensively due to its importance in several proposed devices in logic, sensing, and recording. ${ }^{1,2}$ Physically it is important to understand how to move the domain wall quickly and reliably, which in part depends on the magnetic structure of the domain wall. ${ }^{3-6}$ In a thin, narrow nanowire, the presence of a domain wall is energetically unfavorable so it is necessary to inject the domain wall into the wire. It is known that a domain wall moves the quickest, with a constant structure when driven by magnetic fields weaker than the so-called Walker field, which is typically about 15 Oe in a nanowire. ${ }^{3,7}$ Here we report micromagnetic simulation results describing a technique to quickly inject a domain wall with a controllable magnetic structure into a nanowire with external magnetic field strengths less than the Walker field. The injection technique demonstrates the influence of the topological nature of a domain wall. Controlling the topology of the domain wall makes it possible to reliably inject and densely pack multiple domain walls into a wire, which is necessary for viable magnetic nanowire devices.

The domain wall injection was simulated by integrating the three-dimensional time dependent Landau-Lifshitz equation of motion for a magnetic moment in an applied field. ${ }^{8}$ The materials parameters used are for a permalloylike material $\left(M_{s}=8 \times 10^{5} \mathrm{~A} / \mathrm{m}, A=1.3 \times 10^{-11} \mathrm{~J} / \mathrm{m}\right)$ with a damping constant of 0.008 and a cubic discretization cell size ranging from 2 to $5 \mathrm{~nm}$ on edge. For the simulation results presented here, the nanowire has a rectangular crosssectional area of $100 \times 5 \mathrm{~nm}^{2}$ and the injection pad is a 5 -nm-thick disk with a $500 \mathrm{~nm}$ diameter. The diameter of the pad was kept small so that it would remain single domain as vortices and domain walls inside the pad influence the injection properties. ${ }^{9}$ In each case, a saturating field was applied at $170^{\circ}$ from the $+x$-axis and then slowly relaxed so that the initial magnetic state was primarily in the $-x$ direction. For consistency, each simulated structure was started from the same initial magnetic state. A magnetic field was applied along the long axis of the wire ( $+x$-axis) to inject a head-tohead domain wall along with a bias field applied along the $y$-axis to control the direction of the magnetic moments in

${ }^{a)}$ Electronic mail: andrew.kunz@marquette.edu. the domain wall. The external fields were applied at time zero, and a wall was considered to be injected if it was in the wire after $2 \mathrm{~ns}$. The typical injection time was under a nanosecond and we never found a case where it was likely that waiting longer would result in an injection.

In a thin, narrow nanowire, the strong shape anisotropy determines that the magnetic moments lie in the plane of the wire and are oriented along the long axis. The simplest transition between domains is a transverse wall that separates two head-to-head or tail-to-tail domains. ${ }^{10}$ In a long, straight wire, domain walls can be nucleated at the ends of the wire by a large magnetic field. As shown in Fig. 1, the nucleation field is found to be over 200 Oe for a $100 \times 5 \mathrm{~nm}^{2}$ wire. This nucleation field depends on the dimensions of the wire and the bias field but even as the dimensions are increased the nucleation field is much greater than the Walker field, about 15 Oe in a $100 \times 5 \mathrm{~nm}^{2}$ wire. $^{3,7}$ It is possible to use an injection pad, which is essentially a large magnetic region attached at one end of the wire, to inject domain walls with a lower field from one end of the wire. The pad is typically located with the wire in the middle. ${ }^{11-13}$ The inclusion of a $500 \mathrm{~nm}$ diameter disk decreases the injection field to around 100 Oe. Previous work with the wire offset from the center of the pad shows injection fields of this magnitude. ${ }^{9}$ In Fig. 1 , the injection field is found to be independent of the bias field used to inject domain walls of a known chirality, when the wire is located at the center of the disk. Moving the wire to the top or bottom edge of the disk leads to a sharp reduc-
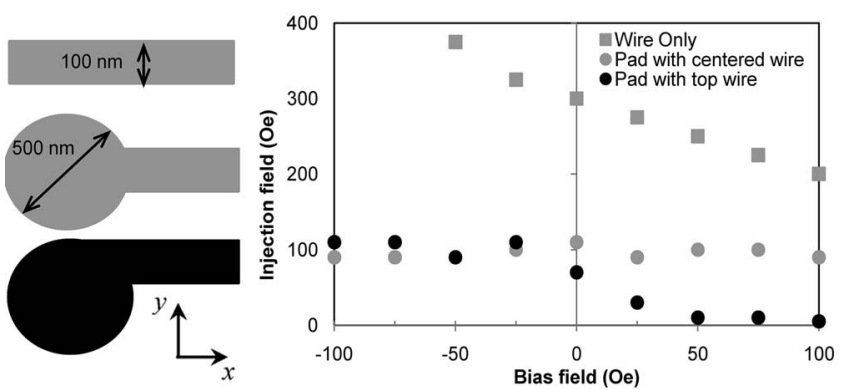

FIG. 1. Injection fields ( $x$-direction) as a function of bias field ( $y$-direction) for three injection geometries into a $100 \times 5 \mathrm{~nm}^{2}$ wire. The injection field is decreased for small positive bias fields when the wire is moved to the top edge of the injection pad. 


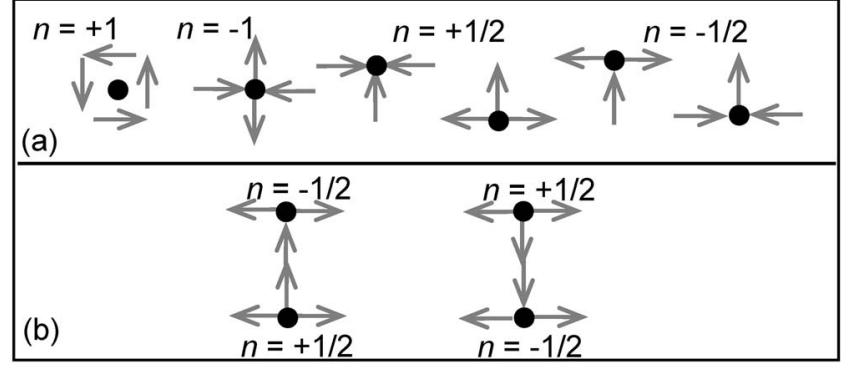

FIG. 2. (a) Cartoon representations of the magnetic structure for 2D topologic defects with their winding numbers $n$. Edge defects have half-integer winding numbers. In (b), two composite representations for a transverse domain wall. The net winding number is zero.

tion in the injection field for reasonable bias fields. As shown in Fig. 1 when the wire is located at the top of the disk, bias fields greater than $50 \mathrm{Oe}$ in the $+y$-direction lower the injection field to as little as 5 Oe. This field is less than the critical Walker field resulting in the fastest, most reliable domain wall motion. ${ }^{3,7}$ Interestingly, the injection field is not symmetric for negative bias fields; there is a strong dependence on the direction of the bias field when the wire is located at an edge. This implies that the injection structures geometry preferentially selects a domain wall orientation. This behavior is confirmed by moving the wire to the bottom edge of the disk. In this case the behavior is reversed; the injection field is small for negative bias fields and large for positive bias fields. The topological structure of a domain wall can be used to explain the selectivity.

Moving the wire from the center of the injection disk to the top reduces the number of corner pinning sites from two to one, however Fig. 1 shows that the injection field is essentially the same without a bias field (and for negative bias fields) for the two disk structures. This behavior implies that a reduction in the number of corner pinning sites is not responsible for the lower injection field but that the pinning mechanism itself is important. Domain wall pinning is a rich topic of current interest due to its importance in reliably moving walls through wires. ${ }^{11,13}$ Domain walls can be pinned by both notches and antinotches. ${ }^{14-16}$ We rely on a topologic model of a domain wall to explain the injection mechanism. A domain wall can be considered a topological object consisting of two or more defects. ${ }^{17}$ In a twodimensional (2D) structure, like a thin wire, the typical defects can be described by a winding number, with a vortex having a winding number of $n=+1$ and an anti-vortex of $n$ $=-1$ as shown in Fig. 2(a). When defects are found along the edge of a wire, the winding numbers are half-integer structures of which several orientations are presented in Fig. 2(a). A domain wall is a composite object of zero net winding number, and we present domain wall orientations for a simple transverse wall in Fig. 2(b).

A simulated time lapse sequence of the injection of a domain wall into a wire is presented in Fig. 3(a). Note that along the top edge of the system the winding number is positive but along the bottom edge it is negative. The domain wall injects in about 700 ps. In Fig. 3(b) and 3(c), cartoon representations of the four possible transverse domain orientations are presented. It is of particular importance to note that when the winding number of the defect along the edge of the system with no pinning sites is positive, the wall easily injects, otherwise it is pinned. It is always the positive defect

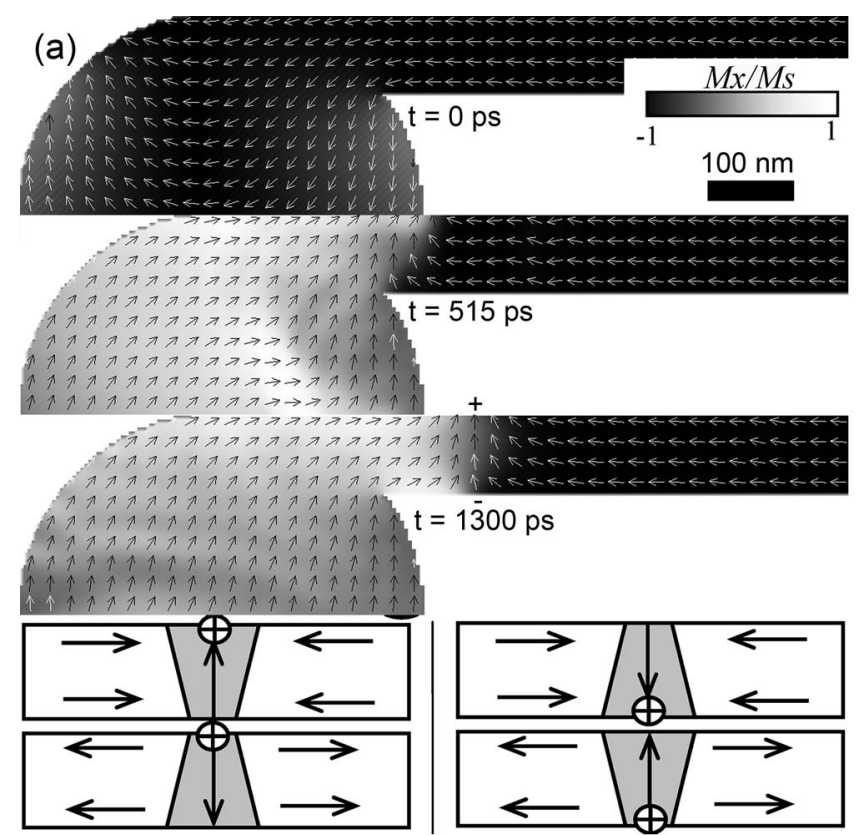

(b) inject easy

(c) inject hard

FIG. 3. (a) Simulated time lapse sequence for the typical injection of a domain wall into the wire with a bias field in the $+y$ direction. The injection pad saturates and the wall is pushed into the wire by the small injection field when positive defects are along the top edge [(a) and (b)]. The domain orientations in (c) have large injection fields because the + defects are pinned at the injection site.

that is pinned. This behavior of the positive defect being pinned is consistent with the behavior noticed in antinotches where negative defects are pinned by notches. . $^{14,16}$

When the wire is located at, or slightly offset from, the center of the pad a positive defect must always pass a pinning site to enter the wire, independent of the domain wall chirality. ${ }^{9}$ By moving the wire to the top of the injection pad, a bias field can be used to arrange the location of the positive defect. This eliminates the domain wall pinning site and the wall easily injects. The dependence on the strength of the bias field is due to the ease of rotating the magnetization of the nanopad. While the bias field rotates the magnetization of the pad and leaves the magnetic state of the wire unaffected, larger bias fields do a more effective job of aligning the magnetization of the pad as shown in Fig. 3(a). The bias field essentially places the domain wall at the injection site and the wall is easily pushed into the wire by the injecting field. A 50 Oe field is sufficient to cause the magnetization of the small disk to essentially saturate; smaller bias fields are not strong enough to position the wall directly at the end of the wire, thus a larger injection field is needed.

It is possible to inject domain walls with small magnetic fields using a "L" shaped bend in a long wire, and this technique is useful for studying single domain walls but $\mathrm{L}$ geometry only allows for a single domain wall to be in the wire. ${ }^{6,16}$ For nanowire devices to be viable, it will be necessary to inject multiple walls and to control their magnetic structure. ${ }^{1}$ The disk structure we present is capable of realizing this behavior. Once the domain wall is injected into the wire, the fields quickly drive the wall along the length of the wire. Because the injection field is less than the critical Walker field, the domain wall maintains its orientation at all times. It is now possible to inject a second domain wall into 


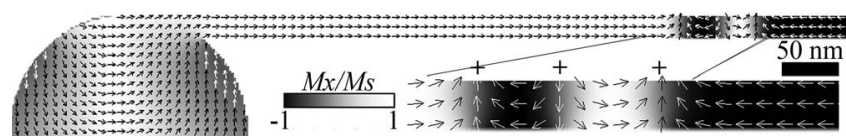

FIG. 4. Simulated image showing three domain walls injected into a $50 \times 5 \mathrm{~nm}$ wire. Due to the orientation of the positive defects and the negative defects, the domain walls do not annihilate when they collide.

the wire by reversing both the bias field and the injection field. The field reversal keeps the winding number of the top defect positive as shown in the cartoon of Fig. 3(b) and a second domain wall quickly is injected. This process can be repeated to inject multiple domain walls into the wire. The simulated injection of three domain walls into a 50 $\times 5 \mathrm{~nm}^{2}$ wire is presented in Fig. 4. The equilibrium domain structure demonstrates the potential to pack many domain walls in a small length of wire. Control of the topological defects allows the domain walls to remain in the wire when the walls collide. ${ }^{17-19}$

In conclusion, a nanodisk is used as a domain wall injector for a magnetic nanowire. When the wire is located at the edge of the nanodisk, the domain walls inject quickly with small fields and a known domain wall orientation. A bias field is used to control the domain wall magnetization but the geometry selects which chirality of domain wall to inject. A simple model using the topological nature of a domain wall was presented to show that controlling the positive defect is necessary to easily inject a domain wall. Control of the injected domain walls leads to a simple method to inject and densely pack multiple walls into a wire.

This work was financially supported by a Cottrell College Science Award from Research Corporation and by the
National Science Foundation (Grant No. DMR-0706194). The authors also acknowledges the course release time supported by the Helen Way Klingler College of Arts and Sciences at Marquette University.

${ }^{1}$ S. S. P. Parkin, M. Hayashi, and L. Thomas, Science 320, 190 (2008).

${ }^{2}$ D. A. Allwood, G. Xiong, C. C. Faulkner, D. Atkinson, D. Petit, and R. P. Cowburn, Science 309, 1688 (2005).

${ }^{3}$ A. Kunz and S. C. Reiff, J. Appl. Phys. 103, $07 D 903$ (2008).

${ }^{4}$ J.-Y. Lee, K.-S. Lee, S. Choi, K. Y. Guslienko, and S.-K. Kim, Phys. Rev. B 76, 184408 (2007).

${ }^{5}$ A. Vanhaverbeke, A. Bischof, and R. Allenspach, Phys. Rev. Lett. 101, 107202 (2008).

${ }^{6}$ E. R. Lewis, D. Petit, A.-V. Jausovec, L. O’Brien, D. E. Read, H. T. Zeng, and R. P. Cowburn, Phys. Rev. Lett. 102, 057209 (2009).

${ }^{7}$ N. L. Schryer and L. R. Walker, J. Appl. Phys. 45, 5406 (1974).

${ }^{8}$ LLG Micromagnetic Simulator (http://llgmicro.home.mindspring.com).

${ }^{9}$ D. McGrouther, S. McVitie, J. N. Chapman, and A. Gentils, Appl. Phys. Lett. 91, 022506 (2007).

${ }^{10}$ R. D. McMichael and M. J. Donahue, IEEE Trans. Magn. 33, 4167 (1997).

${ }^{11}$ C. C. Faulkner, D. A. Allwood, and R. P. Cowburn, J. Appl. Phys. 103, 073914 (2008).

${ }^{12}$ K. Weerts, P. Neutens, L. Lagae, and G. Borghs, J. Appl. Phys. 103, 094307 (2008).

${ }^{13}$ D. Atkinson, D. S. Eastwood, and L. K. Bogart, Appl. Phys. Lett. 92, 022510 (2008).

${ }^{14}$ M. Hayashi, L. Thomas, C. Rettner, R. Moriya, X. Jiang, and S. S. P. Parkin, Phys. Rev. Lett. 97, 207205 (2006).

${ }^{15}$ L. K. Bogart, D. Atkinson, K. O'Shea, D. McGrouther, and S. McVitie, Phys. Rev. B 79, 054414 (2009).

${ }^{16}$ D. Petit, A.-V. Jausovec, D. Read, and R. P. Cowburn, J. Appl. Phys. 103, 114307 (2008).

${ }^{17}$ O. Tchernyshyov and G.-W. Chern, Phys. Rev. Lett. 95, 197204 (2005).

${ }^{18}$ A. Kunz, Appl. Phys. Lett. 94, 132502 (2009).

${ }^{19}$ J.-G. Zhu and Y. Zheng, in Spin Dynamics in Confined Magnetic Structures I, edited by B. Hillebrands and K. Ounadjela (Springer, Berlin, 2002). 